\title{
Hospital Outbreaks Caused by Pseudomonas aeruginosa: Importance of Serogroup O11
}

\section{J. J. FARMER III,${ }^{1+*}$ ROBERT A. WEINSTEIN,$^{2} \ddagger$ C. H. ZIERDT,${ }^{3}$ AND CHARLES D. BROKOPP ${ }^{4} \S$}

Enteric Section ${ }^{1}$ and Hospital Infections Branch, ${ }^{2}$ Centers for Disease Control, Atlanta, Georgia 30333 ; Clinical Pathology Department, Clinical Center, National Institutes of Health, Bethesda, Maryland 202053, and Department of Parasitology and Laboratory Practice, School of Public Health, University of North Carolina, Chapel Hill, North Carolina 27514

Received 12 February 1982/Accepted 3 May 1982

Suspected outbreaks caused by Pseudomonas aeruginosa in 19 hospitals and two motels were studied. On the basis of epidemiological analysis, serological typing, and antibiotic resistance patterns, 17 were classified as single-strain outbreaks. Six were classified as common-source outbreaks: of these, three were caused by contaminated urological instruments or solutions, two involved bathing in contaminated whirlpools, and one was caused by contaminated lens prostheses implanted during eye surgery. The ability of $P$. aeruginosa to survive or grow in wet environments was important in each of these six outbreaks. Eight outbreaks were classified as cross-infection. Two involved the urinary tract and were caused by antibiotic-resistant strains. Six involved the respiratory tract, but only one was caused by an antibiotic-resistant strain. In 2 of the 17 single-strain outbreaks, the exact mode of transmission could not be determined. One was an outbreak of pseudobacteremia in which patient blood cultures were contaminated with a single strain, presumably during collection of specimens or culture processing. $P$. aeruginosa serogroup $\mathrm{O} 11$ caused 9 of 17 (53\%) single-strain outbreaks, a surprising finding since this serogroup represents only about $8 \%$ of endemic hospital isolates of this species. Serotyping was very useful in epidemiological analysis, but antibiotic susceptibility patterns were less useful.

Pseudomonas aeruginosa accounts for about $11 \%$ of all nosocomial infections (4). Although infections caused by $P$. aeruginosa occur sporadically in all hospitals, many occur in clusters (18), suggesting cross-infection or commonsource spread. We studied isolates of $P$. aeruginosa from suspected outbreaks in 21 institutions $(7,9,10,23)$. This report compares serotyping results and antibiograms, the two most common markers used for comparing strains of $P$. aeruginosa ; points out the importance of serogroup O11 in outbreaks; summarizes some of the epidemiological findings; and discusses possible control measures.

\section{MATERIALS AND METHODS}

Microbiology. All isolates of $\boldsymbol{P}$. aeruginosa were originally identified by the submitting laboratory. Isolates that did not have the typical characteristics (blue-

\footnotetext{
† Present address: Nosocomial Infections Laboratory Branch, Hospital Infections Program, Center for Infectious Diseases, Centers for Disease Control, Atlanta, GA 30333.

$\ddagger$ Present address: Infection Control Program, Michael Reese Medical Center, Chicago, IL 60616.

§ Present address: Epidemiology Studies Program, Idaho Department of Health and Welfare, Statehouse, Boise, ID 83720.
}

green pigment, fruity odor, characteristic colonial morphology) were confirmed biochemically at the Enteric Section, Centers for Disease Control (CDC) $(6,7)$. Antimicrobial susceptibility patterns (antibiograms) were determined by the single-disk method of Bauer et al. (3). It is now well accepted that $P$. aeruginosa has intrinsic resistance to most antibiotics and is often susceptible only to colistin, carbenicillin, gentamicin, tobramycin, and amikacin (22). Since all of our isolates were susceptible to colistin, only resistance to the latter four drugs is mentioned. ( $P$. aerugin$o s a$ is also usually susceptible to the third-generation cephalosporins introduced into the United States after this study was completed.) A resistant strain was defined to be one resistant to carbenicillin, gentamicin, tobramycin, or amikacin. Serotyping was done by slide agglutination with live antigens taken from the antibiogram plates and with 17 commercial antisera against the $\mathrm{O}$ antigens of $P$. aeruginosa $(6,7)$. All of these methods have been described in greater detail elsewhere $(6,7,11,20)$.

Outbreaks studied. Unsolicited cultures from possible outbreaks in 21 institutions in the United States (13 states) were submitted to the Enteric Section, CDC, for typing. None had been typed by the sender. Epidemiological information about the outbreak was supplied by infection control personnel, usually both an infection control nurse and a physician-epidemiologist, who did the actual investigation. The final results of the epidemiological investigation were discussed by 
TABLE 1. Summary of common-source outbreaks

\begin{tabular}{|c|c|c|c|c|}
\hline \multirow[b]{2}{*}{ Institution } & \multirow[b]{2}{*}{ Infection site } & \multirow[b]{2}{*}{ Common source } & \multicolumn{2}{|c|}{ P. aeruginosa } \\
\hline & & & Serogroup & Resistant to ${ }^{a}$ : \\
\hline 1. Federal hospital & Urinary tract & Contaminated resectoscope & O4 & CB, GM, TB \\
\hline 2. Community hospital & Urinary tract & Contaminated cystoscope & 06 & CB, GM, TB \\
\hline 3. University hospital & Urinary tract & Contaminated irrigation syringe & 011 & GM \\
\hline 4. Community hospital & Eye & Contaminated lens prosthesis & 06 & $-^{b}$ \\
\hline 5. Motel & Skin & Contaminated whirlpool & O11 & - \\
\hline 6. Motel & Skin & Contaminated whirlpool & O11 & - \\
\hline
\end{tabular}

${ }^{a}$ Only resistance to carbenicillin (CB), gentamicin (GM), tobramycin (TB), or amikacin is listed.

$b$ The strain was susceptible to all four.

telephone with R.A.W., a physician-epidemiologist with the Hospital Infections Branch, CDC. Of the 21 outbreaks, 19 occurred in acute-care hospitals, and 2 were in motels and were associated with bathing in contaminated whirlpools or swimming pools (see Tables 1 and 2). More detailed information about the outbreaks in hospital 4 and motels 5 and 6 has already been published $(9,10,23)$.

\section{RESULTS AND DISCUSSION}

Summary of outbreaks. Routine surveillance in the 21 institutions had shown presumed clustering of Pseudomonas infections of the respiratory tract (seven outbreaks), urinary tract (six outbreaks), blood stream (four outbreaks), skin (two outbreaks), eye (one outbreak), or wounds (one outbreak). On the basis of epidemiological investigations and laboratory results, 17 of the outbreaks were defined as single-strain outbreaks: six were common-source outbreaks, eight were cross-infection outbreaks, one was an outbreak of pseudobacteremia caused by bacterial contamination introduced during specimen collection or culture processing, and two were outbreaks for which the exact mode of transmission could not be determined (caused by $P$. aeruginosa serogroups $\mathrm{O} 11$ and $\mathrm{O6}$ ). Four of the suspect outbreaks were not confirmed to be actual outbreaks.

Common-source outbreaks. In six of the single-strain outbreaks (Table 1), a common source was implicated epidemiologically and verified by culture results and serological typing (institutions 3, 4, 5, and 6) or implicated epidemiologically at a time when adequate cultures of the sources were no longer available (hospitals 1 and 2). The common-source outbreaks in hospitals 1 through 4 involved 6 to 17 patients, with an average of 11 patients. In one common-source outbreak of urinary tract infections (hospital 1), several secondary cases caused by cross-infection from a patient infected with the epidemic strain occurred after the common source had been eliminated.

The three urinary tract outbreaks (Table 1) were caused by antibiotic-resistant strains of three serogroups which had contaminated urological instruments or solutions used at the hos- pital. The other three strains, two of serogroup O11 and one of serogroup O6, were not antibiotic resistant and were not originally derived from a hospital setting (the strain obtained from the hospital 4 outbreak was introduced before the lens reached the hospital [10]). This finding is similar to those for Serratia outbreaks, in which hospital strains are more resistant to antibiotics than are strains originating from outside the hospital (11).

The spread of the common-source infections was facilitated by gross breaks in asepsis, such as the failure to disinfect irrigation equipment (hospital 3), and by the ability of $P$. aeruginosa to survive and actually grow in wet environments $(12,14,26)$, including inappropriate disinfectants such as dilute aqueous benzalkonium chloride (hospitals 1 and 2), swimming pool or whirlpool water (institutions 5 and 6), and bicarbonate suspending fluid used in the packaging of eye lenses (hospital 4). Most common-source outbreaks caused by $P$. aeruginosa can be explained by the ecological adaptation of this organism to grow in wet environments, even those low in nutrients such as disinfectants or distilled water (12).

Cross-infection outbreaks. In eight of the single-strain outbreaks, cross-infection was implicated as the predominant mode of spread (Table 2). Five of the eight outbreaks were caused by $P$. aeruginosa serogroup O11. Most of the crossinfection outbreaks involved the respiratory tract and affected patients who were intubated or had tracheostomies. These outbreaks occurred when in-use respirator tubing was not changed regularly or when aseptic respiratory care technique was not practiced. When these deficiencies were corrected, epidemic spread of $P$. aeruginosa ceased in each outbreak. In one of the outbreaks involving the respiratory tract cross-infection, the epidemic strain was introduced into the respiratory-care unit by a newly admitted patient with chronic lung disease. The outbreak ended when this patient was removed from the unit and the intubated patients were separated from one another.

In two of the eight cross-infection outbreaks 
TABLE 2. Summary of cross-infection outbreaks

\begin{tabular}{|c|c|c|c|}
\hline \multirow[b]{2}{*}{ Institution } & \multirow[b]{2}{*}{ Infection site } & \multicolumn{2}{|c|}{$P$. aeruginosa } \\
\hline & & Serogroup & Resistant to ${ }^{a}$ : \\
\hline 7. University hospital & Urinary tract & O11 & CB, GM, TB \\
\hline 8. Federal hospital & Urinary tract & $\mathrm{O} 12$ & $\mathrm{CB}, \mathrm{GM}$ \\
\hline 9. Municipal hospital & Respiratory tract & $\mathrm{O} 11$ & $-b$ \\
\hline 10. Community hospital & Respiratory tract & O6 & - \\
\hline 11. University hospital & Respiratory tract & O1 & - \\
\hline 12. University hospital & Respiratory tract & $\mathrm{O} 11$ & - \\
\hline 13. Community hospital & Respiratory tract & O11 & GM \\
\hline 14. University hospital & Respiratory tract & O11 & - \\
\hline
\end{tabular}

${ }^{a}$ Only resistance to carbenicillin (CB), gentamicin (GM), tobramycin (TB), or amikacin is listed.

$b$ The strain was susceptible to all four.

(hospitals 8 and 14), infected patients extensively contaminated ambu bags (rubber bags used with endotracheal tubes to inflate the lung), urine testing apparatus, soap, and other items that came into direct contact with patients or hospital personnel. Such contamination resulted in an expanded reservoir for the epidemic strain and in secondary common-source spread. These outbreaks lasted longer (4 and 8 months) and involved more patients (14 and 40) than the other cross-infection outbreaks, which had an average duration of 1 month and involved an average of only six patients.

Outbreak of pseudobacteremia. Hospital 15 had an outbreak of pseudobacteremia caused by $P$. aeruginosa serogroup O1. During 1 week, blood cultures of 17 patients from 7 nursing units on five floors were positive for $P$. aeruginosa. The clinical status and course of 16 of the 17 patients were not compatible with pseudomonas sepsis. The conclusion of the on-site epidemiological investigation was that the blood cultures were contaminated with $P$. aeruginosa serogroup $\mathrm{O} 1$ during collection or processing. The incriminated strain was not found in a culture survey of blood collecting and culture processing equipment done after the outbreak ended. However, other outbreaks of pseudobacteremia have been traced to contaminated commercial vacuum tubes used for collecting blood, contaminated skin antiseptics, and various processing errors (25).

Presumed outbreaks that were not outbreaks. For 4 of the 21 suspect outbreaks, no epidemic strain was found. One of these four outbreaks represented an artifact of surveillance. Systematic review showed that there had been no real change in the infection rate, only an increased interest. In two hospitals, an early attempt was being made to evaluate a potential infection problem in a critical hospital area. Each hospital sent isolates from two infected patients who were in specialty units where pseudomonas infections had been rare and cross-infection or common-source spread of pseudomonas could be disastrous (in one hospital, a prematureinfant intensive-care unit; in the other, a leukemia ward). In both instances, the two strains were of different serogroups and were interpreted as being unrelated. Follow-up in the units confirmed that there were no additional linked cases. For these two hospitals, the serotyping data was of great value since the possibility of cross-infection or a common-source exposure to a virulent strain could not have been excluded on the basis of antibiograms and epidemiological findings. The fourth cluster consisted of four patients with urinary tract infections. The physician-microbiologist at the hospital noted that all four strains of $P$. aeruginosa had mucoid colonies, an unusual finding for strains isolated from urine. No epidemiological relationship among the infected patients could be demonstrated, and all four mucoid strains had different $\mathrm{O}$ antigens.

$P$. aeruginosa serogroup 011 in outbreaks. In a previous study (7) of 425 endemic strains from six hospitals, serogroup $\mathrm{O} 11$ accounted for an average of only $8 \%$ of the endemic isolates (14, $11,10,9,6$, and $0 \%$ ). However, in the present study, this serogroup caused 9 of the 17 singlestrain outbreaks. These were unsolicited cultures from many parts of the United States. There was no further correlation among any of the serotypes and infection site, mode of transmission, or location or type of institution.

The role of $P$. aeruginosa serogroup 011 in causing skin rashes associated with bathing in contaminated pools is now well documented $(9$, $14,16,21,23)$ and is probably related to the distribution of this serogroup in pools (14). 011 is also the predominant serogroup associated with otitis externa in deep-sea divers who stay in large pressure chambers for several weeks (1). However, we were surprised to find so many hospital outbreaks caused by this serogroup. No information was available on the distribution of endemic serogroups of $P$. aeruginosa in any of the 17 institutions. However, some institutions 
included endemic isolates along with epidemic isolates, and only 2 of $42(5 \%)$ endemic isolates were of serogroup O11. These data and the previously published distribution data suggest that serogroup 011 strains occur more frequently in epidemic situations than they do endemically. This finding may be due to any of a number of factors that influence the ability of this group to survive and grow in the hospital environment, resist disinfection, colonize human tissue, invade, and cause disease. Further investigation is needed to learn why $P$. aeruginosa serogroup O11 causes hospital outbreaks.

Evaluation of serotyping and antibiograms as markers of pseudomonas outbreaks. Serotyping is feasible in most clinical microbiology laboratories since antisera are available commercially and the technique can be based on slide agglutination of living cells. Brokopp and co-workers (7) found that about $95 \%$ of the clinical isolates from six hospitals (425 strains) were typable. Almost $90 \%$ of these could be typed with only 9 of the 17 sera. Most strains agglutinate in only one antiserum, and this is a stable property of the cultures. The one drawback to serotyping is that isolates of the more common $\mathrm{O}$ groups may be different strains (in a genetic or epidemiological sense) even though they share the same $O$ antigen. Additional methods such as pyocin typing, bacteriophage susceptibility, and determination of the $\mathrm{H}$ antigen can be used for subdividing strains of the same $O$ group, but none of these methods is practical for routine use. A set of typing sera for $\mathrm{O}$ groups 1 through 12 , which will agglutinate about $90 \%$ of hospital strains, can be kept in a refrigerator almost indefinitely and can be used in the investigation of an infection problem. Unlike most species of $E n$ terobacteriaceae, $P$. aeruginosa has intrinsic resistance to many antibiotics (22). Although resistance to carbenicillin, gentamicin, amikacin, or tobramycin can be very helpful in spotting a possible infection problem (2), several observations indicate that antibiogram comparisons of $\boldsymbol{P}$. aeruginosa should be interpreted cautiously in epidemiological analysis. First, in 11 of the single-strain outbreaks, the antibiogram of the epidemic strain varied, with change in susceptibility to sulfadiazine or carbenicillin accounting for most of the variability. Second, more than $50 \%$ of the epidemic and endemic isolates from 5 of the 11 institutions sending both types of isolates had the same antibiogram. Third, although gentamicin resistance in pseudomonas is often assumed to be a helpful marker, for two outbreaks (hospitals 2 and 3) we found that both epidemic and endemic strains were gentamicin resistant. Resistance to gentamicin was variable in the epidemic strain from one outbreak (hospital 13). These findings are not surprising since it has been shown that endemic strains of $\boldsymbol{P}$. aeruginosa can become resistant to carbenicillin and the aminoglycosides when these drugs are used clinically $(2,8,13,15)$. Only a very resistant antibiogram with high resistance to both carbenicillin and aminoglycosides proved to be a consistent marker. The media used in antibiotic susceptibility testing should be carefully quality controlled to prevent the introduction of an additional variable. The results of $P$. aeruginos $a$ susceptibility to aminoglycoside antibiotics are known to depend on the concentration of cations present.

Others have also noted that the antibiotic resistance pattern of an epidemic strain can change during an outbreak, particularly an outbreak extended in time or space, and that strains that are not related epidemiologically can have the same antibiogram (17). These data indicate that serotyping can be a useful addition to antibiotic susceptibility testing. Moreover, serotyping of $P$. aeruginosa can provide valuable surveillance data in selected specialty units housing high-risk patients and can be useful in the further differentiation of antibiotic-resistant isolates.

Control measures. Ongoing active surveillance of hospital-acquired infections is critical for early detection of outbreaks. Once an outbreak is detected, control measures should be quickly instituted based on prior experience $(5,17,19)$ and thorough, on-site, epidemiological investigation. Control of common-source outbreaks depends on finding and eliminating the contaminated source; however, as we showed, late cross-infections may result once the epidemic organism has been introduced into the patient population.

Pseudomonas cross-infection outbreaks may be more difficult to control, particularly if a large reservoir of asymptomatic infection (or of gastrointestinal or respiratory tract colonization) has been established or if extensive environmental contamination has led to secondary commonsource spread. Measures for controlling crossinfection outbreaks $(5,19,24)$ include the following: identification and appropriate isolation of infected (and at times colonized) patients; physical separation of high-risk patients, such as those with endotracheal tubes or urinary catheters; emphasis on hand washing and aseptic techniques, including the use of gloves when manipulating infected sites; and detection and elimination of any secondary environmental contamination and common-source spread of infection.

Although transient hand carriage of gramnegative bacteria by personnel who have not washed after patient contact is well recognized as a critical step in cross-infection outbreaks, the importance of persistent pseudomonas colo- 
nization of the hospital staff is unknown. In the outbreaks reported here, serological typing was helpful in exonerating and returning to work several hospital workers who were throat or gut carriers of $P$. aeruginosa.

\section{ADDENDUM}

Since the original planned study was completed and our report was prepared, C.H.Z. received unsolicited cultures from seven additional outbreaks (these did not receive the complete epidemiological follow-up of the original study). Six of the seven outbreaks were caused by $P$. aeruginos $a$ serogroup $\mathrm{O} 11$, and the other involved serogroups $\mathrm{O} 11$ and O6. The sources and suspected mode of transmission, as reported by the submitting investigators, were as follows (all outbreaks caused by $P$. aeruginosa serogroup 011 unless otherwise indicated): (i) endocarditis in heroin addicts which was community acquired and caused by contaminated syringes, (ii) respiratory tract infections in a community hospital caused by a contaminated bronchoscope, (iii) urinary tract infections in a community hospital caused by contaminated catheters, (iv) skin rashes at a spa caused by the contaminated water in a swimming pool, $(v)$ postsurgical wound infections at a community hospital intensive-care unit (undetermined mode of transmission), (vi) respiratory tract infections in a community hospital nursery (undetermined mode of transmission), and (vii) respiratory tract infections in a community hospital nursery caused by $P$. aeruginosa serogroups $\mathrm{O} 11$ and $\mathrm{O} 6$ (undetermined mode of transmission).

These data strengthen the association of $P$. aeruginosa $\mathrm{O} 11$ and outbreaks.

\section{LITERATURE CITED}

1. Alcock, S. R. 1977 . Acute otitis externia in divers working in the North Sea: a microbiological survey of seven saturation dives. J. Hyg. 78:395-409.

2. Barnham, M., A. C. Maddocks, and H. Gaya. 1975. Pseudomonas aeruginosa resistant to gentamicin. Lancet i: 576 .

3. Bauer, A. W., W. M. M. Kirby, J. C. Sherris, and M. Turck. 1966. Antibiotic susceptibility testing by a standardized single disc method. Am. J. Clin. Pathol. 45:493496.

4. Bennett, J. V. 1974. Nosocomial infections due to Pseudomonas. J. Infect. Dis. 230(Suppl.):S4-7.

5. Bennett, J. V., and P. S. Brachman (ed.). 1979. Hospital infections. Little Brown and Co., Boston.

6. Brokopp, C. D., and J. J. Farmer III. 1979. Typing methods for Pseudomonas aeruginosa, p. 89-133. In R. G. Doggett (ed.), Pseudomonas aeruginosa. Academic Press, Inc., New York.

7. Brokopp, C. D., R. Gomez-Lus, and J. J. Farmer III. 1977. Serological typing of Pseudomonas aeruginosa: use of commercial antisera and live antigens. J. Clin. Microbiol. 5:640-649.
8. Brown, M. R. W., E. M. Fenton, and W. M. Watkins. 1972. Tetracycline-sensitive/polymyxin-resistant Pseudomonas aeruginosa. Lancet ii:86.

9. Center for Disease Control. 1975. Pool-associated rash illness-North Carolina. Morbid. Mortal. Weekly Rep. 24:349-350.

10. Center for Disease Control. 1976. Endophthalmitis associated with implantation of intra-ocular lens prosthesis. Morbid. Mortal. Weekly Rep. 25:369.

11. Farmer, J. J., III, B. R. Davis, F. W. Hickman, D. B. Presley, G. P. Bodey, M. Negut, and R. A. Bobo. 1976. Detection of Serratia outbreaks in hospital. Lancet ii:455459.

12. Favero, M. S., L. A. Carson, W. W. Bond, and N. J. Peterson. 1971. Pseudomonas aeruginosa: growth in distilled water from hospitals. Science 173:836-838.

13. Hoffman, T. A., and W. E. Bullock. 1970. Carbenicillin therapy of pseudomonas and other gram-negative bacillary infections. Ann. Intern. Med. 73:165-171.

14. Jacobson, J. A., A. W. Hoadley, and J. J. Farmer III. 1976. Pseudomonas aeruginosa serogroup 11 and poolassociated skin rash. Am. J. Public Health 66:1092-1093.

15. Jacoby, G. A. 1974 . Properties of R plasmids determining gentamicin resistance by acetylation in Pseudomonas aeruginosa. Antimicrob. Agents Chemother. 6:239-252.

16. McCausland, R. S., and P. J. Cox. 1975. Pseudomonas infection traced to motel whirlpool. J. Environ. Health 37:455-459.

17. Schaberg, D. R., R. H. Alford, R. Anderson, J. J. Farmer III, M. A. Melly, and W. J. Schaffiner. 1976. An outbreak of nosocomial infection due to multiply resistant Serratia marcescens: evidence of interhospital spread. J. Infect. Dis. 134:181-188.

18. Schaberg, D. R., R. W. Haley, A. K. Highsmith, R. L. Anderson, and J. E. McGowan. 1980. Nosocomial bacteriuria: a prospective study of case clustering and antimicrobial resistance. Ann. Intern. Med. 93:420-424.

19. Schaberg, D. R., R. A. Weinstein, and W. E. Stamm. 1976. Epidemics of nosocomial urinary tract infection caused by multiply resistant gram-negative bacilli: epidemiology and control. J. Infect. Dis. 133:363-366.

20. Stanier, R. Y., N. J. Palleroni, and M. Doudoroff. 1966. The aerobic pseudomonads: a taxonomic study. J. Gen. Micribiol. 43:159-271.

21. Suasker, W. F., J. L. Aeling, M. C. Fitzpatrick, and F. N. Judson. 1978. Pseudomonas folliculitis acquired from a health spa whirlpool. J. Am. Med. Assoc. 239:2362-2365.

22. von Graevinitz, A. 1977. Are microbial identification and sensitivity testing necessary for effective chemotherapy?, p. 169-179. In A. Bondi, J. T. Bartola, and J. E. Prier (ed.), The clinical laboratory as an aid in chemotherapy of infectious disease. University Park Press, Baltimore.

23. Washburn, J., J. A. Jacobson, E. Marson, and B. Thorsen. 1976. Pseudomonas aeruginosa rash with a whirlpool. J. Am. Med. Assoc. 235:2205-2207.

24. Weinstein, R. A., and S. A. Kabins. 1981. Strategies for prevention and control of multiple drug resistant nosocomial infections. Am. J. Med. 70:449-454.

25. Weinstein, R. A., and W. E. Stamm. 1977. Pseudoepidemics in hospital. Lancet ii:862-864.

26. Whitby, J. L., and A. Rampling. 1972. Pseudomonas aeruginosa contamination in domestic and hospital environments. Lancet i:15-17. 